\title{
Estimation of High-Density Activation Maps During Atrial Fibrillation
}

\author{
Alejandro Alcaine ${ }^{1,2}$, Natasja M. S. de Groot ${ }^{3}$, Pablo Laguna ${ }^{1,2}$, \\ Juan Pablo Martínez ${ }^{1,2}$, Richard P. M. Houben ${ }^{4}$ \\ ${ }^{1}$ Biomedical Signal Interpretation and Computational Simulation (BSICoS) Group, Aragón Institute \\ of Engineering Research (I3A), IIS Aragón, Universidad de Zaragoza, Zaragoza, Spain. \\ ${ }^{2}$ CIBER in Bioengineering, Biomaterials and Nanomedicine (CIBER-BBN), Madrid, Spain. \\ ${ }^{3}$ Translational Electrophysiology Unit, Department of Cardiology, Erasmus Medical Center, \\ Rotterdam, The Netherlands. \\ ${ }^{4}$ Applied Biomedical Systems B.V., Maastricht, The Netherlands.
}

\begin{abstract}
The study of activation maps using multi-electrode arrays (MEA) can help to understand atrial fibrillation $(A F)$ mechanisms. Activation mapping based on recorded unipolar electrograms $(u-E G M)$ rely on the local activation time (LAT) detector, which has a limited robustness, accuracy, and generally requires manual post-edition. In general, LAT detection ignores spatiotemporal information about activation and conduction conveyed by the relation between signals of the MEA sensor.

This work proposes an approach to construct activation maps by simultaneous analysis of $u$-EGMs from small clusters of MEA electrodes. The algorithm iteratively fits an activation pattern model to the acquired data. Accuracy was evaluated by comparing with audited maps created by expert electrophysiologists from a patient undergoing open-chest surgery during AF. The estimation error was $-0.29 \pm 6.01$ ms (236 maps, 28369 LATs) with high correlation $(\rho=0.93)$. Therefore, activation maps can be decomposed into local activation patterns derived from fitting an activation model, resulting in smooth and comprehensive high-density activation maps.
\end{abstract}

\section{Introduction}

Atrial fibrillation (AF) is one of the most common arrhythmias, especially in elderly people [1]. Despite the number of proposed mechanisms underlying AF [2-6], the complete phenomena that initiate and perpetuate the arrhythmia is not yet fully understood [4]. Therefore AF treatment is far from being fully effective.

Activation mapping is the most commonly used method for visualization and study of cardiac arrhythmias [7]. During irregular tachycardia like AF, simultaneous mapping is needed due to the non-repetitive nature and complex- ity of the arrhythmia [4]. For high-density simultaneous mapping, a multi-electrode array (MEA) mapping sensor is needed. The propagation of cardiac activation is shown by displaying the recorded signals in a matrix related with the location of the electrodes on the MEA sensor [8]. Detection of local activation times (LAT) for activation mapping is typically associated to the unipolar electrogram (uEGM) steepest negative slope [9]. Reconstruction of activation maps is usually done by combining detected LATs from each of the electrodes on the MEA sensor. However, this procedure ignores the spatiotemporal information embedded in the u-EGM signal.

Cardiac electrophysiological modelling describes the ion currents flowing through the myocardial cell membrane embedded in realistic structures and geometries of the human heart [10]. A less detailed approach (and less time-consuming) represents the cardiac activation propagation as an equivalent source model which uses current sources and densities to calculate the potentials, hence describing the activation propagation as a uniform double layer (UDL) model [11].

In this work, a spatiotemporal approach for estimation and construction of high-density activation maps is presented. The proposed method iteratively fits the UDL activation pattern model to acquired cardiac activity on small clusters of the MEA sensor and reconstructs the complete activation map by combining the solutions for each cluster.

\section{Materials}

The clinical data used in this work belongs to a patient admitted for open chest surgery at Erasmus Medical Center Rotterdam (Rotterdam, The Netherlands) in whom an additional intraoperative electrophysiological study was performed. During the intervention, a custom-made highdensity MEA mapping sensor (Applied Biomedical Sys- 
a)

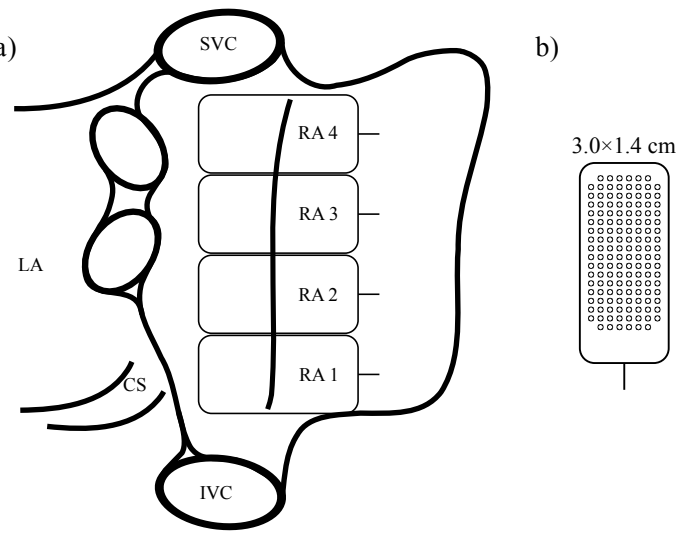

Figure 1. Schematic of the mapping procedure in posterior view: a) Anatomical location of the MEA sensor in the right atrium and b) MEA sensor used for mapping procedure. CS: Coronary Sinus, IVC: Inferior Vena Cava, LA: Left Atrium, RA: Right Atrium, SVC: Superior Vena Cava.

tems B.V., Maastricht, The Netherlands) was sequentially positioned on the epicardial wall of the right atrium, in caudal to cranial direction as shown in Fig. 1(a).

The MEA sensor measures $3.0 \times 1.4 \mathrm{~cm}$ and is composed by 128 circular gold plated electrodes $(2 \mathrm{~mm}$ interelectrode distance, $1 \mathrm{~mm}$ diameter) organized in an $8 \times 16$ rectangular grid. Corner electrodes were not available for recording, resulting in $124 \mathrm{u}-\mathrm{EGM}$ signals for analysis (Fig. 1(b)). The acquired u-EGM signals were band-pass filtered $(1-500 \mathrm{~Hz})$, sampled and digitized at $1 \mathrm{kHz}$. The length of the recorded episodes was of 10s.

Automatic LAT detection was performed off-line [12] and audited by an expert electrophysiologist, hence considered as "ground truth" for performance evaluation.

\section{Methods}

Before processing of the complete map, the mapping array is segmented into 44 overlapping cluster groups of $5 \times 5$ electrodes (area $64 \mathrm{~mm}^{2}$ ), being this the analizing electrode group size of the algorithm.

\subsection{Activation pattern, tissue model and signal generation}

The basic activation pattern can be generalized as a concentrically spreading activation from a focus point with uniform conduction velocity $(\mathrm{CV})$. This activation pattern is applied to a UDL using the boundary element method [11]. A $12 \times 12 \mathrm{~mm}$ slice of atrial tissue with $2 \mathrm{~mm}$ thickness, i.e., the human atrial average thickness [13], is considered. A virtual MEA sensor with $5 \times 5,2 \mathrm{~mm}$ spaced, circular electrodes was placed on the UDL enabling calculation of electrical activity during concentric activation by using the solid angle from a plane triangle formula [11].

\subsection{Conduction velocity measurement and initial location}

Activation focus and $\mathrm{CV}$ are the basic parameters to define a concentric activation pattern. Those parameters must to be estimated from acquired data.

Thus, CV is estimated by a process inspired in that presented by Fitzgerald et al. [14]. This process consist on adjusting a biquadratic surface in the least square sense [15] to the time delay, computed as those maximizing the cross-covariance between the signal at each of the electrodes and that of the central electrode from the $5 \times 5$ group under analysis.

This CV is estimated for each $5 \times 5$ group of electrodes, remaining unmodified during the rest of the analysis. After that, the proposed iterative algorithm searches for the focus location that generates the concentric activation. Hence the algorithm needs to be initialized in an appropriate location. This initial location $\mathbf{f}_{0}$ is established as the coordinates of the electrode $i_{\text {min }}$ which matches the following joint minimization criteria:

$$
i_{\text {min }}=\underset{i}{\arg \min }\left\{\delta_{i}+R_{\mathrm{RS}}^{i}\right\},
$$

where $\delta_{i}$ is the time delay of the $i$ th electrode to the reference electrode and $R_{\mathrm{RS}}^{i}$ stands for the R-to-S ratio of the $i$ th $\mathrm{u}-\mathrm{EGM}$ signal [16]. Therefore, the initial position $\mathbf{f}_{0}$ correspond to that from the electrode coordinates with earlier activation and lower R-to-S difference.

\subsection{Iterative algorithm and map recon- struction}

The iterative algorithm aims to optimize the fitting of the concentric propagation model to the recording data by modifying the focus location of the propagation pattern in test. The algorithm starts from the previous determined initial location $\mathbf{f}_{0}$. This location is updated for each $k$ th iteration, $k=1 \ldots 50$, following:

$$
\mathbf{f}_{k+1}=\mathbf{f}_{k}+\Delta \cdot \mathbf{u}_{k},
$$

where $\Delta$ represents the updating step and $\mathbf{u}_{k}$ is the unitary update vector in the direction that improves the matching between the modelled and recorded u-EGMs. In this work, update step $\Delta$ is set up to the measured $\mathrm{CV}$ value, so that the focus moves in each iteration a distance equivalent to the wavefront propagation in $1 \mathrm{~ms}$, i.e., the signal resolution of the acquired data.

The unitary vector $\mathbf{u}_{k}$ is driven by the comparison of the acquired u-EGM signals with the derived u-EGM signals 


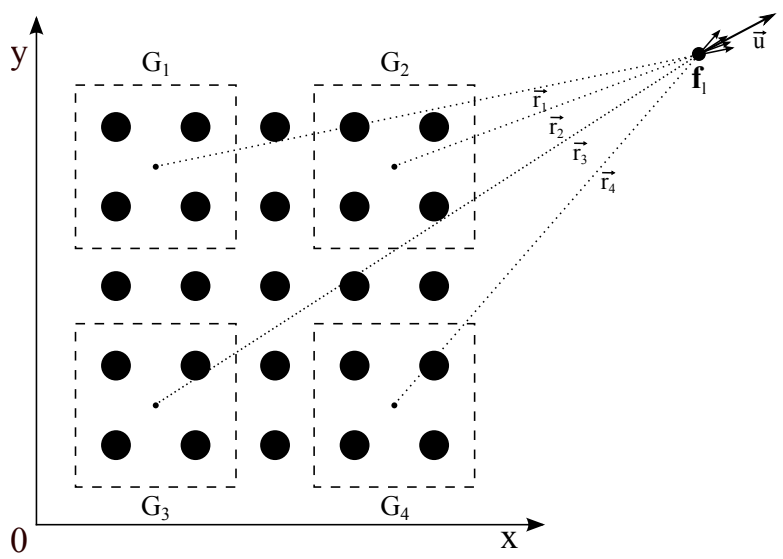

Figure 2. Diagram of the focus movement algorithm shown on a $5 \times 5$ group of electrodes (big dots). Dashed squares shown the $2 \times 2$ subgroup of electrodes $\mathrm{G}_{d}, d=$ $1 \ldots 4$ and their direction vector $\mathbf{r}_{d}$ to the activation focus $\mathbf{f}_{l}$. Vector $\mathbf{u}_{k}$ stands for the update vector for the next iteration. Note: Characters with arrows appear boldface in text.

at iteration $k$. The time difference in the activation time between acquired and modelled u-EGM signals for each $i$ th electrode is obtained by maximizing the cross-covariance function:

$$
\tau_{i}=\underset{m}{\arg \max }\left\{C_{i}[m]\right\},
$$

where $C_{i}[m]$ stands for the cross-covariance function between the acquired and modelled signals at lag $m$ for the $i$ th electrode of the current $5 \times 5$ analysis group. Hence a median group delay $\tilde{\tau}_{d}, d=1 . .4$, is obtained from each $2 \times 2$ subgroup of electrodes $\mathrm{G}_{d}$ located at the corner of the $5 \times 5$ group under analysis, as illustrated in Fig. 2. A unitnorm director vector $\mathbf{r}_{d}$ from the center of each $\mathrm{G}_{d}$ to the current focus location is identified and the update vector is obtained as:

$$
\boldsymbol{u}_{k}=\sum_{d=1}^{4} \frac{\tilde{\tau}_{d}}{\sum_{d}\left|\tilde{\tau}_{d}\right|} \mathbf{r}_{d},
$$

which its unitary form $\mathbf{u}_{k}$ is used in (2). This process is repeated until a cost function $Q$ exceeds a threshold or the maximum number of $k$ iterations are reached. The cost function $Q$ is defined as:

$$
Q=\operatorname{mean}_{i}\left\{C_{i}[0]\right\} .
$$

After complete analysis of the $445 \times 5$ groups of electrodes conforming the $8 \times 16$ MEA sensor, the activation sequence is obtained by weighted averaging the individual LAT estimates for each solution using the final value of the cost function $Q$ as weights.
Table 1. Error (mean \pm SD) and Spearman's correlation coefficient $\rho$ comparing the estimated LATs with the manual reference.

\begin{tabular}{lcccc}
\hline \hline Location & $\begin{array}{c}\text { Maps } \\
(\#)\end{array}$ & $\begin{array}{c}\text { LATs } \\
(\#)\end{array}$ & $\begin{array}{c}\text { Error } \\
(\mathrm{ms})\end{array}$ & $\rho$ \\
\hline$R A 1$ & 51 & 5751 & $0.43 \pm 10.65$ & $0.81^{*}$ \\
$R A 2$ & 61 & 7471 & $0.50 \pm 3.77$ & $0.95^{*}$ \\
$R A 3$ & 60 & 7351 & $-1.00 \pm 3.42$ & $0.96^{*}$ \\
$R A 4$ & 64 & 7796 & $-0.91 \pm 4.56$ & $0.96^{*}$ \\
\hline All & 236 & 28369 & $-0.29 \pm 6.01$ & $0.93^{*}$ \\
\hline \hline
\end{tabular}

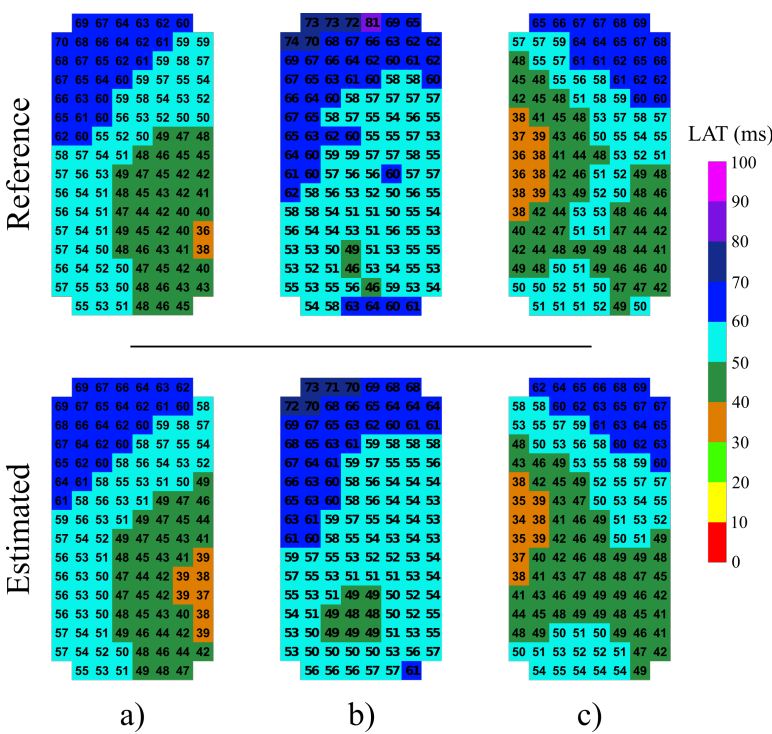

Figure 3. Examples of activation map reconstruction: a) A single wavefront passing through the MEA sensor, b) breakthrough activation event happening behind the MEA sensor and c) two wavefront coming from different parts of the MEA sensor and colliding behind the MEA sensor. LATs are colour-coded from earliest (red colour) to latest (pink colour) activated electrode in $10 \mathrm{~ms}$ isochrone steps.

\section{Results}

Evaluation was done by comparing reconstructed maps with those obtained by an expert electrophysiologist. Error is presented as mean \pm standard deviation (SD). Since LATs have an ordered nature, Spearman's correlation $\rho$ was assessed and a $p$-value $\leqslant 0.05$ was required for statistical significance.

Table 1 summarizes the detection results obtained per each recording location. A total of 236 maps (beats) were analyzed with an error of $-0.29 \pm 6.01 \mathrm{~ms}$ with high Spearman's correlation $(\rho=0.93, p<0.01)$. Figure 3 show representative examples of map reconstruction. 


\section{Discussion and conclusion}

This work introduces a spatiotemporal detection approach that allows to obtain smooth and comprehensive high-density activation maps. A simplified activation pattern and conduction model are used in order to synthesize u-EGM signals that mimic the measured activation map using an iterative process.

Results show high detection accuracy compared with audited annotations in the right atrium during AF. However, this work is a preliminary study of application on a single patient and atrial location data.

Although this work uses invasive data, its results suggest that the proposed algorithm can be used to study highdensity activations maps and in the development of minimally invasive epicardial high-density mapping.

\section{Acknowledgements}

This study was supported by personal grants to A.A. refs.: BES-2011-046644 and EEBB-I-13-06613, by project TEC2013-42140-R from MINECO. Also by Aragón Government (Spain) and European Social Fund (EU) through Grupo Consolidado BSICoS ref.: T96. The CIBER-BBN is an initiative of Instituto de Salud Carlos III.

\section{References}

[1] Fuster V, Rydén LE, Cannom DS, Crijns HJ, Curtis AB, Ellenbogen KA, Halperin JL, Le Heuzey JY, Kay GN, Lowe JE, Olsson SB, Prystowsky EN, Tamargo JL, Wann S. ACC/AHA/ESC 2006 guidelines for the management of patients with atrial fibrillation-executive summary. Journal of the American College of Cardiology 2006;48(4):854 -906 .

[2] Moe G, Abildskov J. Atrial fibrillation as a self-sustaining arrhythmia independent of focal discharge. American Heart Journal 1959;58(1):59 - 70.

[3] Haïssaguerre M, Jaïs P, Shah DC, Takahashi A, Hocini M, Quiniou G, Garrigue S, Le Mouroux A, Le Métayer P, Clémenty J. Spontaneous initiation of atrial fibrillation by ectopic beats originating in the pulmonary veins. New England Journal of Medicine 1998;339(10):659 - 666.

[4] Eckstein J, Kuhne M, Osswald S, Schotten U. Mapping of atrial fibrillation-basic research and clinical applications. Swiss Medical Weekly 2009;139(35-36):496 - 504.

[5] Lee G, Kumar S, Teh A, Madry A, Spence S, Larobina M, Goldblatt J, Brown R, Atkinson V, Moten S, Morton JB, Sanders P, Kistler PM, Kalman JM. Epicardial wave mapping in human long-lasting persistent atrial fibrillation: transient rotational circuits, complex wavefronts, and disorganized activity. European Heart Journal 2014;35(2):8697.

[6] de Groot NMS, Houben RPM, Smeets JL, Boersma E, Schotten U, Schalij MJ, Crijns H, Allessie MA. Electropathological substrate of longstanding persistent atrial fibrillation in patients with structural heart disease: Epicardial breakthrough. Circulation 2010;122(17):1674 - 1682.

[7] Yaksh A, Kik C, Knops P, Roos-Hesselink J, Bogers A, Zijlstra F, Allessie MA, De Groot NMS. Atrial fibrillation: to map or not to map? Netherlands Heart Journal 2013;1 - 8 .

[8] Konings KT, Kirchhof CJ, Smeets JR, Wellens HJ, Penn OC, Allessie MA. High-density mapping of electrically induced atrial fibrillation in humans. Circulation 1994; 89(4): 1665 - 1680.

[9] Spach MS, Miller WT, Miller-Jones E, Warren RB, Barr RC. Extracellular potentials related to intracellular action potentials during impulse conduction in anisotropic canine cardiac muscle. Circulation Research 1979;45(2):188 204.

[10] Dössel O, Krueger M, Weber F, Wilhelms M, Seemann G. Computational modeling of the human atrial anatomy and electrophysiology. Medical and Biological Engineering and Computing 2012;50(8):773 - 799 .

[11] van Oosterom A. Solidifying the solid angle. Journal of Electrocardiology 2002;35(4, Part B):181 - 192.

[12] Houben RPM, de Groot NMS, Allessie MA. Analysis of fractionated atrial fibrillation electrograms by wavelet decomposition. IEEE Transactions on Biomedical Engineering 2010;57(6): 1388 - 1398 .

[13] Harrild DM, Henriquez CS. A computer model of normal conduction in the human atria. Circulation Research 2000; 87(7):e25 - e36.

[14] Fitzgerald T, Rhee E, Brooks D, Triedman J. Estimation of cardiac conduction velocities using small data sets. Annals of Biomedical Engineering 2003;31(3):250 - 261.

[15] Bayly P, KenKnight B, Rogers J, Hillsley R, Ideker R, Smith W. Estimation of conduction velocity vector fields from epicardial mapping data. IEEE Transactions on Biomedical Engineering 1998;45(5):563 - 571.

[16] Houben RPM, de Groot NMS, Smeets JLRM, Becker AE, Lindemans FW, Allessie MA. S-wave predominance of epicardial electrograms during atrial fibrillation in humans: Indirect evidence for a role of the thin subepicardial layer. Heart Rhythm 2004;1(6):639 - 647.

Address for correspondence:

Alejandro Alcaine Otín

C/ Mariano Esquillor S/N, Edificio I+D+i, L 4.0.05

50018 Zaragoza, Spain.

E-mail: aalcaineo@unizar.es 